\title{
Stable invariant manifolds with impulses and growth rates
}

Lijun $\operatorname{Pan}^{*}$

\section{"Correspondence:} plj1977@126.com

School of Mathematics, Jia Ying University, Meizhou, Guangdong 514015, P.R. China

\section{Springer}

\begin{abstract}
This paper is devoted to the study of stable and unstable behaviors with growth rates $e^{c \rho(t)}$ for impulsive differential equations, where $\rho(t)$ is some increasing continuous function. By the techniques of impulsive analysis, we obtain the existence of stable invariant manifolds for the impulsive perturbed equation provided that the linear equation admits a $\rho$-nonuniform exponential dichotomy and $f, g$ are sufficiently small Lipschitz perturbation. We also consider the case of exponential contraction and show that the asymptotic stability persists under sufficiently small nonlinear perturbations. In addition, we study how the manifolds vary with the perturbations.
\end{abstract}

MSC: 34D35

Keywords: impulsive differential equation; nonuniform exponential dichotomy; stable invariant manifolds

\section{Introduction}

The theory of impulsive differential equations is attracting much attention in recent years. This is mostly because impulsive differential equations efficiently describe many phenomena arising in engineering, physics, and science as well (see e.g. [1, 2]). We consider the class of impulsive linear equations in $R^{n}$, given by

$$
\left\{\begin{array}{l}
x^{\prime}=A(t) x, \quad t \geq 0, t \neq \tau_{i}, \\
\triangle x\left(\tau_{i}\right)=B_{i} x, \quad i=1,2, \ldots,
\end{array}\right.
$$

where $A(t)$ is a $n \times n$ matrix such that the function $t \rightarrow A(t)$ has at most discontinuities of the first kind at the points $\tau_{i}, B_{i}, i=1,2 \ldots$ are $n \times n$ matrices, and the impulsive times $0<\tau_{1}<\tau_{2}<\cdots$ satisfy $\lim _{i \rightarrow \infty} \tau_{i}=\infty, \Delta x\left(\tau_{i}\right)=x\left(\tau_{i}^{+}\right)-x\left(\tau_{i}^{-}\right), x\left(\tau_{i}^{+}\right)=\lim _{h \rightarrow 0^{+}} x\left(\tau_{i}+h\right)$, $x\left(\tau_{i}^{-}\right)=\lim _{h \rightarrow 0^{-}} x_{i}\left(\tau_{i}+h\right), i=1,2, \ldots$ By a solution $x$ of (1), we mean a real function on $[0, \infty)$ of class $C^{1}$ outside the points $\tau_{i}$ such that $x$ is left-continuous (thus, $\Delta x\left(\tau_{i}\right)=x\left(\tau_{i}^{+}\right)-$ $\left.x\left(\tau_{i}\right)\right)$, and $x$ satisfies $x^{\prime}(t)=A(t) x(t)$ at each point $t \in[0, \infty)$ with the possible exception of the points $t=\tau_{i}$ and $\triangle x\left(\tau_{i}\right)=B_{i} x$ for $i=1,2, \ldots$. We always assume that (1) has a unique solution and all solutions of (1) are global.

It is well known that the notion of exponential dichotomy, going back to Perron in [3], plays an important role in invariant manifolds of differential equation. The theory of the exponential dichotomy and its applications are widely developed. We can refer to the books [4-6] for details and references. There are a lot of linear differential equations with

(c) 2015 Pan. This article is distributed under the terms of the Creative Commons Attribution 4.0 International License (http://creativecommons.org/licenses/by/4.0/), which permits unrestricted use, distribution, and reproduction in any medium, provided you give appropriate credit to the original author(s) and the source, provide a link to the Creative Commons license, and indicate if changes were made. 
exponential dichotomy. For example, Sacker and Sell [7-10] investigated sufficiently conditions for the existence of exponential dichotomy, also in the infinite-dimensional setting. In [11], Barreira and Valls discussed the much weaker notion of a nonuniform exponential dichotomy. In [12], the authors obtained the integral conditions for a nonuniform polynomial dichotomy. This theory of nonuniform hyperbolicity goes back to the landmark works of Oseledets [13]. For detailed expositions of parts of nonuniform hyperbolicity theory, we can refer to [14].

We note that the study of robustness in the case of uniform exponential behavior has a long history. For example, Massera and Schäffer [15] discussed robustness of uniform exponential behavior in Banach spaces. Perron [3], and Dale'ckiǐ and Krein [5] also studied the robust stability of solution of differential equation under uniform behavior. For more recent work we refer to [16-18]. In particular, in [16], Barreira and Valls studied the existence of stable invariant manifold for the linear equation under any sufficiently small nonlinear perturbation $f$.

However, there are few papers that consider the case of nonuniform behavior for impulsive dynamical systems. For instance, we refer the reader to the works of Barreira and Valls. For example, in [19], they studied the existence of nonuniform exponential dichotomy, and obtained sharp lower and upper bound for the regularity coefficient. In [20], the existence of invariant stable manifolds is established under sufficiently small perturbations of a linear equation and they showed that the invariant manifolds are also of class $C^{1}$ outside the impulsive points. In [21], the authors studied invariant manifolds for impulsive equations under nonuniform polynomial dichotomies. The existence and robustness of nonuniform $(h, k, \mu, v)$-dichotomies for nonautonomous impulsive differential equations are obtained in [22].

In the recent years, general stable and unstable behaviors with growth rates of the form $e^{c \rho(t)}$ for a function $\rho(t)$ are exhibited by Barreira and Valls [23-25]. This type of behavior is called a $\rho$-nonuniform exponential dichotomy. A linear equation with this general asymptotic behavior may have all Lyapunov exponents zero or all Lyapunov exponents infinite besides the usual case $\rho(t)=t$. For example, in [23] the authors showed that for $\rho$ in a large class of rate functions, any linear equation (1) in a finite-dimensional space has a $\rho$-nonuniform exponential dichotomy. In [25], they showed that a $\rho$-nonuniform exponential dichotomy is robust under sufficiently small linear perturbations.

Our main aims in the present paper are as follows:

(1) We establish the existence of invariant stable manifolds for the impulsive perturbed equations

$$
\left\{\begin{array}{l}
x^{\prime}=A(t) x+f(t, x), \quad t \geq 0, t \neq \tau_{i}, \\
\triangle x\left(\tau_{i}\right)=B_{i} x+g_{i}\left(\tau_{i}, x\right), \quad i=1,2, \ldots
\end{array}\right.
$$

where $f: R^{+} \times R^{n} \rightarrow R^{n}$ is a piecewise continuous function in $t \geq 0$ at most with discontinuous of the first kind at the time $\tau_{i}$ satisfying $f(t, 0)=0$ and $g_{i}: R^{+} \times R^{n} \rightarrow R^{n}, i=1,2, \ldots$, are continuous functions satisfying $g_{i}(t, 0)=0$. To obtain the desired results, some techniques of impulsive analysis and a careful control of impulsive times over the whole proof are considered because impulses create some technical difficulties.

(2) We study the behavior of the manifolds under the perturbations showing that the manifolds vary with the perturbations $f$ and $g$. 
The organization of this paper is as follows. In Section 2, we show that the asymptotic stability of a $\rho$-nonuniform exponential contraction persists under sufficiently small nonlinear perturbations. In Section 3, we establish stable manifolds theorem under sufficiently small perturbation of nonuniform exponential dichotomy. We also study the behavior of the manifolds under the perturbations in Section 4, Finally, an example is given to illustrate the applicability of the results in Section 5.

\section{Stability for $\rho$-nonuniform exponential contractions}

In this section, we show that the asymptotic stability of a $\rho$-nonuniform exponential contraction persists under sufficiently small nonlinear perturbations. We recall the notion of a $\rho$-nonuniform exponential contraction.

Let $T(t, s)$ be the linear operator satisfying $x(t)=T(t, s) x(s)$ for any solution of (1) and each $t, s \geq 0$. Consider an increasing function $\rho: R^{+} \rightarrow R^{+}$with $\rho(0)=0$ and $\rho(t) \rightarrow \infty$ as $t \rightarrow \infty$, we say that (1) admits a $\rho$-nonuniform exponential contraction, if for some constants $\mu<0, a \geq 0$, and $D \geq 1$, we have

$$
\|T(t, s)\| \leq D e^{\mu(\rho(t)-\rho(s))+a \rho(s)}, \quad t \geq s \geq 0
$$

We assume that the following conditions hold:

$\left(\mathrm{H}_{1}\right)$ there exist functions $b(t) \geq 0, c(t) \geq 0$ such that for $t \geq 0, x, y \in R^{n}$,

$$
\|f(t, x)-f(t, y)\| \leq b(t)\|x-y\|
$$

and

$$
\left\|g_{i}(t, x)-g_{i}(t, y)\right\| \leq c(t)\|x-y\|
$$

$\left(\mathrm{H}_{2}\right) \quad 0<M=\sup _{s \geq 0}\left\{\int_{s}^{\infty} b(\tau) e^{a \rho(\tau)} d \tau\right\}<\infty ;$

$\left(\mathrm{H}_{3}\right) \Upsilon=\sup _{s \geq 0}\left\{\sum_{\tau_{i} \geq s} c\left(\tau_{i}\right) e^{a \rho\left(\tau_{i}\right)}\right\}<\infty$.

For example, taking $a=1, \rho(t)=t, b(t)=c(t)=\frac{1}{10} e^{-2 t}, \tau_{i}=i, i=1,2, \ldots$, we obtain

$$
M=\sup _{s \geq 0}\left\{\int_{s}^{\infty} b(\tau) e^{a \rho(\tau)} d \tau\right\}=\sup _{s \geq 0}\left\{\frac{1}{10} \int_{s}^{\infty} e^{-\tau} d \tau\right\}=\frac{1}{10}
$$

and

$$
\Upsilon=\sup _{s \geq 0}\left\{\sum_{\tau_{i} \geq s} c\left(\tau_{i}\right) e^{a \rho\left(\tau_{i}\right)}\right\}=\sup _{s \geq 0}\left\{\frac{1}{10} \sum_{i \geq s} e^{-i}\right\}=\frac{1}{10(e-1)} .
$$

Theorem 1 Assume that (1) admits a $\rho$-nonuniform exponential contraction and $\left(\mathrm{H}_{1}\right)$ $\left(\mathrm{H}_{3}\right)$ hold. If

$$
D(M+\Upsilon) \leq \frac{1}{2}
$$

then there exists a unique solution $x(t)$ of $(2)$ with $x(s)=\xi \in R^{n}$ such that

$$
\|x(t)\| \leq \frac{D}{1-D(M+\Upsilon)} e^{\mu(\rho(t)-\rho(s))+a \rho(s)}\|\xi\|
$$

for $s \geq 0, t \geq s$. 
Proof We consider a complete metric space $\mathcal{C}$ of functions $x:[s,+\infty) \rightarrow R^{n}$ such that

(1) $x(s)=\xi$;

(2) $x$ is continuous outside of the points $\tau_{i}$;

(3) $x(t)$ is left-continuous for each $\tau_{i}$;

(4) for $\forall x_{1}=x_{1}(t), x_{2}=x_{2}(t) \in \mathcal{C}$, the distance $d\left(x_{1}, x_{2}\right)$ is defined by

$$
d\left(x_{1}, x_{2}\right)=\sup \left\{\frac{\left\|x_{1}(t)-x_{2}(t)\right\|}{\|\xi\| e^{\mu(\rho(t)-\rho(s))+a \rho(s)}}: t \geq s \geq 0\right\}
$$

(5) $\forall x \in \mathcal{C},\|x\|^{\prime}=\sup \left\{\frac{\|x(t)\|}{\|\xi\| e^{\mu(\rho(t)-\rho(s)+a \rho(s)}}: t \geq s \geq 0\right\} \leq 2 D$.

Set

$$
J(x)(t)=T(t, s) \xi+\int_{s}^{t} T(t, \tau) f(\tau, x(\tau)) d \tau+\sum_{s \leq \tau_{i}<t} T\left(t, \tau_{i}^{+}\right) g_{i}\left(\tau_{i}, x\left(\tau_{i}\right)\right),
$$

for each $x \in \mathcal{C}$ and $t \geq s$. Clearly, $(J x)(s)=\xi$. By $\left(\mathrm{H}_{1}\right)$, for each $x_{1}, x_{2} \in \mathcal{C}$, we have

$$
\left\|f\left(\tau, x_{1}(\tau)\right)-f\left(\tau, x_{2}(\tau)\right)\right\| \leq b(\tau) e^{\mu(\rho(\tau)-\rho(s))+a \rho(s)} d\left(x_{1}, x_{2}\right)
$$

and for $i=1,2, \ldots$

$$
\left\|g_{i}\left(\tau_{i}, x_{1}\left(\tau_{i}\right)\right)-g_{i}\left(\tau_{i}, x_{2}\left(\tau_{i}\right)\right)\right\| \leq c\left(\tau_{i}\right) e^{\mu\left(\rho\left(\tau_{i}\right)-\rho(s)\right)+a \rho(s)} d\left(x_{1}, x_{2}\right) .
$$

Therefore, it follows from (3), (6), (7), $\left(\mathrm{H}_{2}\right)$, and $\left(\mathrm{H}_{3}\right)$ that

$$
\begin{aligned}
& \left\|J\left(x_{1}\right)(t)-J\left(x_{2}\right)(t)\right\| \\
& \leq \int_{s}^{t}\|T(t, \tau)\|\left\|f\left(\tau, x_{1}(\tau)\right)-f\left(\tau, x_{2}(\tau)\right)\right\| d \tau \\
& \quad+\sum_{s \leq \tau_{i}<t}\left\|T\left(t, \tau_{i}^{+}\right)\right\|\left\|g_{i}\left(\tau_{i}, x_{1}\left(\tau_{i}\right)\right)-g_{i}\left(\tau_{i}, x_{2}\left(\tau_{i}\right)\right)\right\| \\
& \leq D e^{\mu(\rho(t)-\rho(s))+a \rho(s)}\|\xi\| d\left(x_{1}, x_{2}\right)\left[\int_{s}^{t} b(\tau) e^{a \rho(\tau)} d \tau+\sum_{s \leq \tau_{i}<t} c\left(\tau_{i}\right) e^{a \rho\left(\tau_{i}\right)}\right] \\
& \leq D e^{\mu(\rho(t)-\rho(s))+a \rho(s)}(M+\Upsilon)\|\xi\| d\left(x_{1}, x_{2}\right) .
\end{aligned}
$$

Thus we have

$$
d\left(J\left(x_{1}\right)(t), J\left(x_{2}\right)(t)\right) \leq D(M+\Upsilon) d\left(x_{1}, x_{2}\right) .
$$

By (4), the operator $J$ becomes a contraction. Furthermore, by (3), we get

$$
\begin{aligned}
\|J(x)\| & \leq\|T(\cdot, s) \xi\|+\int_{s}^{t}\|T(t, \tau)\|\|f(\tau, x(\tau))\| d \tau+\sum_{s \leq \tau_{i}<t}\left\|T\left(t, \tau_{i}^{+}\right)\right\|\left\|g_{i}\left(\tau_{i}, x\left(\tau_{i}\right)\right)\right\| \\
& \leq D e^{\mu(\rho(t)-\rho(s))+a \rho(s)}\|\xi\|+D(M+\Upsilon) e^{\mu(\rho(t)-\rho(s))+a \rho(s)}\|\xi\| d(x, 0) .
\end{aligned}
$$


Thus

$$
\|J(x)\|^{\prime} \leq D+D(M+\Upsilon)\|x\|^{\prime} \leq D+\frac{1}{2}\|x\|^{\prime} \leq 2 D,
$$

which shows that $J(\mathcal{C}) \subset \mathcal{C}$. Therefore, there exists a unique function $x \in \mathcal{C}$ such that $J x=x$. By (10), we have $\|x\| \leq \frac{D}{1-D(M+\Upsilon)} e^{\mu(\rho(t)-\rho(s))+a \rho(s)}\|\xi\|$. This completes the proof of the theorem.

\section{Stable invariant manifolds}

This section is devoted to establishing our stable manifolds theorem under a sufficiently small perturbation of nonuniform exponential dichotomy.

We say that (1) admits a $\rho$-nonuniform exponential dichotomy if there exist projections $P(t)$ for $t \geq 0$ satisfying

$$
P(t) T(t, s)=T(t, s) P(s), \quad t, s \geq 0,
$$

and there exist constants $\mu<0 \leq v, a \geq 0$, and $D \geq 1$ such that for each $t \geq s \geq 0$

$$
\|T(t, s) P(s)\| \leq D e^{\mu(\rho(t)-\rho(s))+a \rho(s)}, \quad\left\|T(t, s)^{-1} Q(t)\right\| \leq D e^{-v(\rho(t)-\rho(s))+a \rho(t)},
$$

where $Q(t)=I d-P(t)$. Set the linear subspaces

$$
E(t)=P(t)\left(R^{n}\right) \quad \text { and } \quad F(t)=Q(t)\left(R^{n}\right)
$$

and let $\Phi$ be the space of functions $\phi:[0, \infty) \times R^{n} \rightarrow R^{n}$ having at most discontinuities of the first kind in the first variable such that for each $s \geq 0$ and $x, y \in E(s), \phi(s, 0)=0$, $\phi(s, E(s)) \subset F(s)$ and

$$
\|\phi(s, \xi)-\phi(s, \bar{\xi})\| \leq\|\xi-\bar{\xi}\|, \quad \xi, \bar{\xi} \in E(s)
$$

with the distance

$$
d(\phi, \varphi)=\sup \{\|\phi(s, \xi)-\psi(s, \xi)\| /\|\xi\|: s \geq 0, \xi \in E(s)\}
$$

Obviously, $\Phi$ becomes a complete metric space. Given a sequence $\phi \in \Phi$, we consider the set

$$
\mathcal{W}=\{(s, \xi, \phi(s, \xi)): s \geq 0, \xi \in E(s)\}
$$

Assume that (1) admits a $\rho$-nonuniform exponential dichotomy, the unique piecewise continuous solution $(x(t), y(t)) \in E(t) \times F(t)$ of (2) with initial condition $(\xi, \eta) \in E(s) \times F(s)$ satisfies

$$
\begin{aligned}
x(t)= & T(t, s) \xi+\int_{s}^{t} P(t) T(t, \tau) f(\tau, x(\tau), y(\tau)) d \tau \\
& +\sum_{s \leq \tau_{i}<t} P(t) T\left(t, \tau_{i}^{+}\right) g_{i}\left(\tau_{i}, x\left(\tau_{i}\right), y\left(\tau_{i}\right)\right)
\end{aligned}
$$


and

$$
\begin{aligned}
y(t)= & T(t, s) \eta+\int_{s}^{t} Q(t) T(t, \tau) f(\tau, x(\tau), y(\tau)) d \tau \\
& +\sum_{s \leq \tau_{i}<t} Q(t) T\left(t, \tau_{i}^{+}\right) g_{i}\left(\tau_{i}, x\left(\tau_{i}\right), y\left(\tau_{i}\right)\right) .
\end{aligned}
$$

We consider the semiflow

$$
\Psi_{r}(s, \xi, \eta)=(s+r, x(s+r), y(s+r)), \quad r \geq 0
$$

which is generated by (2). To obtain the desired results, we also need the following conditions:

$\left(\mathrm{H}_{4}\right) \quad 0<\gamma=\sup _{i \geq 1}\left\{\frac{\ln \left(1+2 D c\left(\tau_{i}\right) e^{a \rho\left(\tau_{i}\right)}\right)}{\rho\left(\tau_{i}\right)-\rho\left(\tau_{i-1}\right)}\right\}<\infty ;$

$\left(\mathrm{H}_{5}\right) \quad 0<\bar{M}=\sup _{s \geq 0}\left\{e^{(a+\nu-\mu) \rho(s)} \int_{s}^{\infty} b(\tau) e^{(\mu-\nu+a+\gamma) \rho(\tau)} d \tau\right\}<\infty ;$

$\left(\mathrm{H}_{6}\right) \quad 0<\bar{\Upsilon}=\sup _{s \geq 0}\left\{e^{(a+\nu-\mu) \rho(s)} \sum_{\tau_{i} \geq s} c\left(\tau_{i}\right) e^{(\mu-v+a+\gamma) \rho\left(\tau_{i}\right)}\right\}<\infty$.

Theorem 2 Assume that (1) admits a $\rho$-nonuniform exponential dichotomy and $\left(\mathrm{H}_{1}\right)-\left(\mathrm{H}_{6}\right)$ hold. If

$$
\begin{aligned}
& D(M+\Upsilon) \leq \frac{1}{4}, \\
& \mu-v+a<0, \\
& 2 D^{2} e^{2 D M}(\bar{M}+\bar{\Upsilon}) \leq 1,
\end{aligned}
$$

and

$$
\left[\frac{D^{2}}{1-2 D(M+\Upsilon)}+\frac{2 D^{2} e^{2 D M}(M+\Upsilon)}{1-2 D(M+\Upsilon)}\right](\bar{M}+\bar{\Upsilon})<1
$$

then there exists a unique function $\phi \in \Phi$ such that

$$
\Psi_{r}(s, \xi, \phi(s, \xi)) \in \mathcal{W}
$$

for $r \geq 0$. Furthermore, for every $s \geq 0, \xi, \bar{\xi} \in E(s)$ and $r=t-s \geq 0$

$$
\left\|\Psi_{r}(s, \xi, \phi(s, \xi))-\Psi_{r}(s, \bar{\xi}, \phi(s, \bar{\xi}))\right\| \leq 2 D e^{2 D M} e^{(\mu+\gamma)(\rho(t)-\rho(s))+(a+\gamma) \rho(s)}\|\xi-\bar{\xi}\| .
$$

Proof We replace (14) and (15) by

$$
\begin{aligned}
x(t)= & T(t, s) x(s)+\int_{s}^{t} P(t) T(t, \tau) f(\tau, x(\tau), \phi(\tau, x(\tau))) d \tau \\
& +\sum_{s \leq \tau_{i}<t} P(t) T\left(t, \tau_{i}^{+}\right) g_{i}\left(\tau_{i}, x\left(\tau_{i}\right), \phi\left(\tau_{i}, x\left(\tau_{i}\right)\right)\right)
\end{aligned}
$$


and

$$
\begin{aligned}
\phi(t, x(t))= & T(t, s) \phi(s, x(s)) \xi+\int_{s}^{t} Q(t) T(t, \tau) f(\tau, x(\tau), \phi(\tau, x(\tau))) d \tau \\
& +\sum_{s \leq \tau_{i}<t} Q(t) T\left(t, \tau_{i}^{+}\right) g_{i}\left(\tau_{i}, x\left(\tau_{i}\right), \phi\left(\tau_{i}, x\left(\tau_{i}\right)\right)\right) .
\end{aligned}
$$

Next, we show some lemmas to finish our proof of the theorem.

Lemma 1 Given $\xi \in E(s)$, for every $\phi \in \Phi$, there is a unique piecewise continuous function $x=x_{\phi}:[s,+\infty) \times E(s) \rightarrow R^{n}$ satisfying $(20)$ and

$$
\left\|x_{\phi}(t, \xi)\right\| \leq \frac{D}{1-2 D(M+\Upsilon)} e^{\mu(\rho(t)-\rho(s))+a \rho(s)}\|\xi\|,
$$

for $t \geq s, s \geq 0$.

Proof Given $s \geq 0, \xi \in E(s)$, we consider the operator $\bar{J}$ defined by

$$
\begin{aligned}
\bar{J}(x)(t, \xi)= & T(t, s) \xi+\int_{s}^{t} P(t) T(t, \tau) f(\tau, x(\tau, \xi), \phi(\tau, x(\tau, \xi))) d \tau \\
& +\sum_{s \leq \tau_{i}<t} P(t) T\left(t, \tau_{i}^{+}\right) g_{i}\left(\tau_{i}, x\left(\tau_{i}, \xi\right), \phi\left(\tau_{i}, x\left(\tau_{i}, \xi\right)\right)\right)
\end{aligned}
$$

in the space $\mathcal{A}$ of piecewise continuous functions $x:[s,+\infty) \times E(s) \rightarrow R^{n}$ such that $x(s, \xi)=$ $\xi$ and $d(x, 0) \leq 2 D$, where $d\left(x_{1}, x_{2}\right)$ is given by (5). For every $x, y \in \mathcal{A}$ and $t \geq s$, by $\left(\mathrm{H}_{1}\right)$ and (12), we obtain

$$
\begin{aligned}
& \|f(\tau, x(\tau, \xi), \phi(\tau, x(\tau, \xi)))-f(\tau, y(\tau, \xi), \phi(\tau, y(\tau, \xi)))\| \\
& \quad \leq 2 b(\tau) e^{\mu(\rho(\tau)-\rho(s))+a \rho(s)} d(x(\tau, \xi), y(\tau, \xi))
\end{aligned}
$$

and for $i=1,2, \ldots$

$$
\begin{aligned}
& \left\|g_{i}\left(\tau_{i}, x\left(\tau_{i}, \xi\right), \phi\left(\tau_{i}, x\left(\tau_{i}, \xi\right)\right)\right)-g\left(\tau_{i}, y\left(\tau_{i}, \xi\right), \phi\left(\tau_{i}, y\left(\tau_{i}, \xi\right)\right)\right)\right\| \\
& \quad \leq 2 c\left(\tau_{i}\right) e^{\mu\left(\rho\left(\tau_{i}\right)-\rho(s)\right)+a \rho(s)} d\left(x\left(\tau_{i}, \xi\right), y\left(\tau_{i}, \xi\right)\right) .
\end{aligned}
$$

By (24), (25), and (11), we have

$$
\begin{aligned}
& \|J(x)(t)-J(y)(t)\| \\
& \leq \int_{s}^{t}\|P(t) T(t, \tau)\|\|f(\tau, x(\tau, \xi), \phi(\tau, x(\tau, \xi)))-f(\tau, y(\tau, \xi), \phi(\tau, y(\tau, \xi)))\| d \tau \\
& \quad+\sum_{s \leq \tau_{i}^{<<}}\left\|T\left(t, \tau_{i}^{+}\right)\right\|\left\|g_{i}\left(\tau_{i}, x\left(\tau_{i}, \xi\right), \phi\left(\tau_{i}, x\left(\tau_{i}, \xi\right)\right)\right)-g\left(\tau_{i}, y\left(\tau_{i}, \xi\right), \phi\left(\tau_{i}, y\left(\tau_{i}, \xi\right)\right)\right)\right\| \\
& \leq 2 D e^{\mu(\rho(t)-\rho(s))+a \rho(s)}\|x-y\|^{\prime}\left[\int_{s}^{t} b(\tau) e^{a \rho(\tau)} d \tau+\sum_{s \leq \tau_{i}<t} c\left(\tau_{i}\right) e^{a \rho\left(\tau_{i}\right)}\right] \\
& \leq 2 D(M+\Upsilon) e^{\mu(\rho(t)-\rho(s))+a \rho(s)} d(x, y) .
\end{aligned}
$$


Therefore

$$
d(J(x), J(y)) \leq 2 D(M+\Upsilon) d(x, y) .
$$

The rest of the proof is as in Theorem 1, here we omit it.

Lemma 2 Let $x: R^{+} \rightarrow R^{+}$be a piecewise continuous function at most with discontinuities of the first kind at the points $\tau_{i}$. If

$$
x(t) \leq c+\int_{t_{0}}^{t} m(\tau) x(\tau) d \tau+\sum_{t_{0} \leq \tau_{i}<t} d_{i} x\left(\tau_{i}\right), \quad t \geq t_{0},
$$

for some constants $c>0, d_{i} \geq 0$ and some function $m: R^{+} \rightarrow R^{+}$, then

$$
x(t) \leq c \prod_{t_{0} \leq \tau_{i}<t}\left(1+d_{i}\right) \exp \int_{t_{0}}^{t} m(\tau) d \tau, \quad t \geq t_{0} .
$$

Lemma 3 For every $\phi \in \Phi$, and $\xi, \bar{\xi} \in E(s)$

$$
\left\|x_{\phi}(t, \xi)-x_{\phi}(t, \bar{\xi})\right\| \leq D \exp (2 D M) e^{(\mu+\gamma)(\rho(t)-\rho(s))+(a+\gamma) \rho(s)}\|\xi-\bar{\xi}\|, \quad t \geq s \geq 0 .
$$

Proof Letting $\left\|x_{\phi}(t, \xi)-x_{\phi}(t, \bar{\xi})\right\|^{*}=\frac{\left\|x_{\phi}(t, \xi)-x_{\phi}(t, \bar{\xi})\right\|}{e^{\mu(\rho(t)-\rho(s))+a \rho(s)}}$, by $\left(\mathrm{H}_{1}\right)$ and (12), for $\tau \geq s$, we have

$$
\begin{aligned}
q_{1}(\tau) & =\left\|f\left(\tau, x_{\phi}(\tau, \xi), \phi\left(\tau, x_{\phi}(\tau, \xi)\right)\right)-f\left(\tau, x_{\phi}(\tau, \bar{\xi}), \phi\left(\tau, x_{\phi}(\tau, \bar{\xi})\right)\right)\right\| \\
& \leq 2 b(\tau)\left\|x_{\phi}(\tau, \xi)-x_{\phi}(\tau, \bar{\xi})\right\| \\
& \leq 2 b(\tau) e^{\mu(\rho(\tau)-\rho(s))+a \rho((s)}\left\|x_{\phi}(\tau, \xi)-x_{\phi}(\tau, \bar{\xi})\right\|^{*},
\end{aligned}
$$

and for $i=1,2, \ldots$

$$
\begin{aligned}
q_{2}\left(\tau_{i}\right) & =\left\|g_{i}\left(\tau_{i}, x_{\phi}\left(\tau_{i}, \xi\right), \phi\left(\tau_{i}, x_{\phi}\left(\tau_{i}, \xi\right)\right)\right)-g_{i}\left(\tau_{i}, x_{\phi}\left(\tau_{i}, \bar{\xi}\right), \phi\left(\tau_{i}, x_{\phi}\left(\tau_{i}, \bar{\xi}\right)\right)\right)\right\| \\
& \leq 2 c\left(\tau_{i}\right)\left\|x_{\phi}\left(\tau_{i}, \xi\right)-x_{\phi}\left(\tau_{i}, \bar{\xi}\right)\right\| \\
& \leq 2 c\left(\tau_{i}\right) e^{\mu\left(\rho\left(\tau_{i}\right)-\rho(s)\right)+a \rho(s)}\left\|x_{\phi}\left(\tau_{i}, \xi\right)-x_{\phi}\left(\tau_{i}, \bar{\xi}\right)\right\|^{*}
\end{aligned}
$$

By (28), (29), and (11), we have

$$
\begin{aligned}
&\left\|x_{\phi}(\tau, \xi)-x_{\phi}(\tau, \bar{\xi})\right\| \\
& \leq\|T(t, s) P(s)\|\|\xi-\bar{\xi}\|+\int_{s}^{t}\|T(t, \tau) P(\tau)\| q_{1}(\tau) d \tau+\sum_{s \leq \tau_{i}<t}\left\|T\left(t, \tau_{i}^{+}\right) P\left(\tau_{i}^{+}\right)\right\| q_{2}\left(\tau_{i}\right) \\
& \leq D e^{\mu(\rho(t)-\rho(s))+a \rho(s)}\|\xi-\bar{\xi}\| \\
&+2 D e^{\mu(\rho(t)-\rho(s))+a \rho(s)} \int_{s}^{t} b(\tau) e^{a \rho(\tau))}\left\|x_{\phi}(\tau, \xi)-x_{\phi}(\tau, \bar{\xi})\right\|^{*} d \tau \\
&+2 D e^{\mu(\rho(t)-\rho(s))+a \rho(s)} \sum_{s \leq \tau_{i}^{<}<t} c\left(\tau_{i}\right) e^{a \rho\left(\tau_{i}\right)}\left\|x_{\phi}\left(\tau_{i}, \xi\right)-x_{\phi}\left(\tau_{i}, \bar{\xi}\right)\right\|^{*}
\end{aligned}
$$


Thus

$$
\begin{aligned}
\left\|x_{\phi}(\tau, \xi)-x_{\phi}(\tau, \bar{\xi})\right\|^{*} \leq & D\|\xi-\bar{\xi}\|+2 D \int_{s}^{t} b(\tau) e^{a \rho(\tau))}\left\|x_{\phi}(\tau, \xi)-x_{\phi}(\tau, \bar{\xi})\right\|^{*} d \tau \\
& +2 D \sum_{s \leq \tau_{i}<t} c\left(\tau_{i}\right) e^{a \rho\left(\tau_{i}\right)}\left\|x_{\phi}\left(\tau_{i}, \xi\right)-x_{\phi}\left(\tau_{i}, \bar{\xi}\right)\right\|^{*}
\end{aligned}
$$

By Lemma 2, we have

$$
\begin{aligned}
& \left\|x_{\phi}(t, \xi)-x_{\phi}(t, \bar{\xi})\right\|^{*} \\
& \quad \leq D \prod_{s \leq \tau_{i}<t}\left[1+2 D c\left(\tau_{i}\right) e^{a \rho\left(\tau_{i}\right)}\right] \exp \left[2 D \int_{s}^{t} b(\tau) e^{a \rho(\tau)} d \tau\right]\|\xi-\bar{\xi}\|
\end{aligned}
$$

Let $\tau_{i 1}, \tau_{i 2}, \ldots, \tau_{i l}$ be impulsive points in $[s, t)$. In view of $\left(\mathrm{H}_{4}\right)$, we have

$$
\begin{aligned}
& \prod_{s \leq \tau_{i}<t}\left[1+2 D c\left(\tau_{i}\right) e^{a \rho\left(\tau_{i}\right)}\right] \\
& \quad=\left[1+2 D c\left(\tau_{i 1}\right) e^{a \rho\left(\tau_{i 1}\right)}\right]\left[1+2 D c\left(\tau_{i 2}\right) e^{a \rho\left(\tau_{i 2}\right)}\right] \cdots\left[1+2 D c\left(\tau_{i l}\right) e^{a \rho\left(\tau_{i l}\right)}\right] \\
& \leq e^{\gamma\left(\rho\left(\tau_{i 1}\right)-\rho\left(\tau_{i 1-1}\right)\right)} e^{\gamma\left(\rho\left(\tau_{i 2}\right)-\rho\left(\tau_{i 1}\right)\right)} \cdots e^{\gamma\left(\rho\left(\tau_{i l}\right)-\rho\left(\tau_{i(l-1)}\right)\right)} \\
& =e^{\gamma\left(\rho\left(\bar{\tau}_{i l}\right)-\rho\left(\tau_{i 1-1}\right)\right)} \leq e^{\gamma \rho(t)}
\end{aligned}
$$

where $t_{i 1-1}$ is the first impulsive point before $t_{i 1}$ and satisfies $t_{i 1-1} \leq s$. Thus, it follows that

$$
\left\|x_{\phi}(t, \xi)-x_{\phi}(t, \bar{\xi})\right\| \leq D e^{2 D M} e^{(\mu+\gamma)(\rho(t)-\rho(s))+(a+\gamma) \rho(s)}\|\xi-\bar{\xi}\|
$$

This completes the proof.

Lemma 4 For every $\phi, \psi \in \Phi$ and $\xi \in E(s), t \geq s$,

$$
\left\|x_{\phi}(t, \xi)-x_{\psi}(t, \xi)\right\| \leq \frac{D e^{2 D M}(M+\Upsilon)}{1-2 D(M+\Upsilon)} e^{(\mu+\gamma)(\rho(t)-\rho(s))+(a+\gamma) \rho(s)}\|\xi\| d(\phi, \psi) .
$$

Proof Letting $\left\|x_{\phi}(t, \xi)-x_{\psi}(t, \xi)\right\|^{* *}=\frac{\left\|x_{\phi}(t, \xi)-x_{\psi}(t, \xi)\right\|}{\|\xi\| e^{\mu(\rho(t)-\rho(s))+a \rho(s)}}$, by $\left(\mathrm{H}_{1}\right),(12)$, and Lemma 1, for each $\tau \geq s$, we have

$$
\begin{aligned}
q_{3}(\tau)= & \left\|f\left(\tau, x_{\phi}(\tau, \xi), \phi\left(\tau, x_{\phi}(\tau, \xi)\right)\right)-f\left(\tau, x_{\psi}(\tau, \xi), \psi\left(\tau, x_{\psi}(\tau, \xi)\right)\right)\right\| \\
\leq & b(\tau) \|\left(x_{\phi}(\tau, \xi)-x_{\psi}(\tau, \xi), \phi\left(\tau, x_{\phi}(\tau, \xi)\right)-\psi\left(\tau, x_{\psi}(\tau, \xi)\right) \|\right. \\
\leq & b(\tau)\left[\left\|x_{\phi}(\tau, \xi)-x_{\psi}(\tau, \xi)\right\|+\left\|\phi\left(\tau, x_{\phi}(\tau, \xi)\right)-\psi\left(\tau, x_{\psi}(\tau, \xi)\right)\right\|\right] \\
\leq & b(\tau)\left[\|\left(x_{\phi}(\tau, \xi)-x_{\psi}(\tau, \xi)\|+\| \phi\left(\tau, x_{\phi}(\tau, \xi)\right)-\psi\left(\tau, x_{\phi}(\tau, \xi)\right) \|\right.\right. \\
& \left.+\left\|\psi\left(\tau, x_{\phi}(\tau, \xi)\right)-\psi\left(\tau, x_{\psi}(\tau, \xi)\right)\right\|\right] \\
\leq & b(\tau)\left[\left\|x_{\phi}(\tau, \xi)\right\| d(\phi, \psi)+2\left\|x_{\phi}(\tau, \xi)-x_{\psi}(\tau, \xi)\right\|\right] \\
\leq & \frac{D}{1-2 D(M+\Upsilon)} b(\tau) e^{\mu(\rho(\tau)-\rho(s))+a \rho(s)}\|\xi\| d(\phi, \psi) \\
& +2 b(\tau) e^{\mu(\rho(\tau)-\rho(s))+a \rho(s)}\|\xi\|\left\|x_{\phi}(\tau, \xi)-x_{\psi}(\tau, \xi)\right\|^{* *}
\end{aligned}
$$


and for $i=1,2, \ldots$

$$
\begin{aligned}
q_{4}\left(\tau_{i}\right)= & \left\|g_{i}\left(x_{\phi}\left(\tau_{i}, \xi\right), \phi\left(\tau_{i}, x_{\phi}\left(\tau_{i}, \xi\right)\right)\right)-g_{i}\left(x_{\psi}\left(\tau_{i}, \xi\right), \psi\left(\tau_{i}, x_{\psi}\left(\tau_{i}, \xi\right)\right)\right)\right\| \\
\leq & \frac{D}{1-2 D(M+\Upsilon)} c\left(\tau_{i}\right) e^{\mu\left(\rho\left(\tau_{i}\right)-\rho(s)\right)+a \rho(s)}\|\xi\| d(\phi, \psi) \\
& +2 c\left(\tau_{i}\right) e^{\mu\left(\rho\left(\tau_{i}\right)-\rho(s)\right)+a \rho(s)}\|\xi\|\left\|x_{\phi}\left(\tau_{i}, \xi\right)-x_{\psi}\left(\tau_{i}, \xi\right)\right\|^{* *} .
\end{aligned}
$$

By (36), (37), and (11), we obtain

$$
\begin{aligned}
& \left\|x_{\phi}(t, \xi)-x_{\psi}(t, \xi)\right\| \\
& \leq \int_{s}^{t}\|T(t, \tau) P(\tau)\| q_{3}(\tau) d \tau+\sum_{s \leq \tau_{i}<t}\left\|T\left(t, \tau_{i}^{+}\right) P\left(\tau_{i}^{+}\right)\right\| q_{4}\left(\tau_{i}\right) \\
& \leq \frac{D(M+\Upsilon)}{1-2 D(M+\Upsilon)} e^{\mu(\rho(t)-\rho(s))+a \rho(s)}\|\xi\| d(\phi, \psi) \\
& \quad+2 D e^{\mu(\rho(t)-\rho(s))+a \rho(s)}\|\xi\| \int_{s}^{t} b(\tau) e^{a \rho(\tau)}\left\|x_{\phi}(\tau, \xi)-x_{\psi}(\tau, \xi)\right\|^{* *} d \tau \\
& \quad+2 D e^{\mu(\rho(t)-\rho(s))+a \rho(s)}\|\xi\| \sum_{s \leq \tau_{i}<t} c\left(\tau_{i}\right) e^{a \rho\left(\tau_{i}\right)}\left\|x_{\phi}\left(\tau_{i}, \xi\right)-x_{\psi}\left(\tau_{i}, \xi\right)\right\|^{* *} .
\end{aligned}
$$

Then

$$
\begin{aligned}
\left\|x_{\phi}(t, \xi)-x_{\psi}(t, \xi)\right\|^{* *} \leq & \frac{D(M+\Upsilon)}{1-2 D(M+\Upsilon)} d(\phi, \psi) \\
& +2 D \int_{s}^{t} b(\tau) e^{a \rho(\tau)}\left\|x_{\phi}(\tau, \xi)-x_{\psi}(\tau, \xi)\right\|^{* *} d \tau \\
& +2 D \sum_{s \leq \tau_{i}<t} c\left(\tau_{i}\right) e^{a \rho\left(\tau_{i}\right)}\left\|x_{\phi}\left(\tau_{i}, \xi\right)-x_{\psi}\left(\tau_{i}, \xi\right)\right\|^{* *} .
\end{aligned}
$$

By Lemma 2 and $\left(\mathrm{H}_{4}\right)$, we have

$$
\begin{aligned}
\left\|x_{\phi}(t, \xi)-x_{\psi}(t, \xi)\right\|^{\prime} & \leq \frac{D e^{2 D M}(M+\Upsilon)}{1-2 D(M+\Upsilon)} \prod_{s \leq \tau_{i}<t}\left(1+2 D c\left(\tau_{i}\right) e^{a \rho\left(\tau_{i}\right)}\right)\|\phi-\psi\| \\
& \leq \frac{D e^{2 D M}(M+\Upsilon)}{1-2 D(M+\Upsilon)} e^{\gamma \rho(t)}\|\xi\| d(\phi, \psi),
\end{aligned}
$$

which implies that inequality (35) holds.

Lemma 5 Given $\phi \in \Phi$, the following properties hold:

(1) If for every $s \geq 0, \xi \in E(s), t \geq s$,

$$
\begin{aligned}
\phi\left(t, x_{\phi}(t, \xi)\right)= & T(t, s) \phi(s, \xi)+\int_{s}^{t} T(t, \tau) Q(\tau) f\left(\tau, x_{\phi}(t, \xi), \phi\left(\tau, x_{\phi}(\tau, \xi)\right)\right) d \tau \\
& +\sum_{s \leq \tau_{i}<t} T\left(t, \tau_{i}^{+}\right) Q\left(\tau_{i}^{+}\right) g_{i}\left(\tau_{i}, x_{\phi}\left(\tau_{i}, \xi\right), \phi\left(\tau_{i}, x_{\phi}\left(\tau_{i}, \xi\right)\right)\right)
\end{aligned}
$$


then for every $s \geq 0, \xi \in E(s)$,

$$
\begin{aligned}
\phi(s, \xi)= & -\int_{s}^{\infty} T(\tau, s)^{-1} Q(\tau) f\left(\tau, x_{\phi}(t, \xi), \phi\left(\tau, x_{\phi}(\tau, \xi)\right)\right) d \tau \\
& -\sum_{s \leq \tau_{i}} T\left(\tau_{i}^{+}, s\right)^{-1} Q\left(\tau_{i}^{+}\right) g_{i}\left(\tau_{i}, x_{\phi}\left(\tau_{i}, \xi\right), \phi\left(\tau_{i}, x_{\phi}\left(\tau_{i}, \xi\right)\right)\right) .
\end{aligned}
$$

(2) If identity (42) holds for each $s \geq 0, \xi \in E(s)$, then (41) holds for every $s \geq 0, \xi \in E(s)$, and $t \geq s$.

Proof By $\left(\mathrm{H}_{1}\right),(12)$, and Lemma 1, we have

$$
\begin{aligned}
& \left\|f\left(\tau, x_{\phi}(t, \xi), \phi\left(\tau, x_{\phi}(\tau, \xi)\right)\right)\right\| \\
& \quad \leq 2 b(\tau)\left\|x_{\phi}(\tau, \xi)\right\| \leq \frac{2 D}{1-2 D(M+\Upsilon)} e^{\mu(\rho(\tau)-\rho(s))+a \rho(s)} b(\tau)\|\xi\|,
\end{aligned}
$$

and for $i=1,2, \ldots$

$$
\begin{aligned}
& \left\|g_{i}\left(\tau_{i}, x_{\phi}\left(\tau_{i}, \xi\right), \phi\left(\tau_{i}, x_{\phi}\left(\tau_{i}, \xi\right)\right)\right)\right\| \\
& \quad \leq 2 c\left(\tau_{i}\right)\left\|x_{\phi}\left(\tau_{i}, \xi\right)\right\| \leq \frac{2 D}{1-2 D(M+\Upsilon)} e^{\mu\left(\rho\left(\tau_{i}\right)-\rho(s)\right)+a \rho(s)} c\left(\tau_{i}\right)\|\xi\| .
\end{aligned}
$$

Hence, by $\left(\mathrm{H}_{5}\right)$ and $\left(\mathrm{H}_{6}\right)$, we see that

$$
\begin{aligned}
\int_{s}^{\infty} & T(\tau, s)^{-1} Q(\tau) f\left(\tau, x_{\phi}(t, \xi), \phi\left(\tau, x_{\phi}(\tau, \xi)\right)\right) d \tau \\
\quad \leq & \int_{s}^{\infty}\left\|T(\tau, s)^{-1} Q(\tau)\right\|\left\|f\left(\tau, x_{\phi}(t, \xi), \phi\left(\tau, x_{\phi}(\tau, \xi)\right)\right)\right\| d \tau \\
\quad \leq & \frac{2 D^{2}}{1-2 D(M+\Upsilon)}\|\xi\| e^{(a-\mu+v) \rho(s)} \int_{s}^{\infty} b(\tau) e^{(\mu-v+a) \rho(\tau)} d \tau<\infty
\end{aligned}
$$

and

$$
\begin{aligned}
& \sum_{s \leq \tau_{i}} T\left(\tau_{i}^{+}, s\right)^{-1} Q\left(\tau_{i}^{+}\right) g_{i}\left(\tau_{i}, x_{\phi}\left(\tau_{i}, \xi\right), \phi\left(\tau_{i}, x_{\phi}\left(\tau_{i}, \xi\right)\right)\right) \\
& \quad \leq \sum_{s \leq \tau_{i}}\left\|T\left(\tau_{i}^{+}, s\right)^{-1} Q\left(\tau_{i}^{+}\right)\right\|\left\|g_{i}\left(\tau_{i}, x_{\phi}\left(\tau_{i}, \xi\right), \phi\left(\tau_{i}, x_{\phi}\left(\tau_{i}, \xi\right)\right)\right)\right\| \\
& \quad \leq \frac{2 D^{2}}{1-2 D(M+\Upsilon)}\|\xi\| e^{(a-\mu+v) \rho(s)} \sum_{s \leq \tau_{i}} c\left(\tau_{i}\right) e^{(\mu-v+a) \rho\left(\tau_{i}\right)}<\infty .
\end{aligned}
$$

Therefore, the right-hand side of (42) is well defined. We assume that (41) holds for each $s \geq 0, \xi \in E(s)$, and $t \geq s$, then it follows that

$$
\begin{aligned}
\phi(s, \xi)= & T(t, s)^{-1} Q(t) \phi\left(t, x_{\phi}(t, \xi)\right)-\int_{s}^{t} T(\tau, s)^{-1} Q(\tau) f\left(\tau, x_{\phi}(\tau, \xi), \phi\left(\tau, x_{\phi}(\tau, \xi)\right)\right) d \tau \\
& -\sum_{s \leq \tau_{i}<t} T\left(\tau_{i}^{+}, s\right)^{-1} Q\left(\tau_{i}^{+}\right) g_{i}\left(\tau_{i}, x_{\phi}\left(\tau_{i}, \xi\right), \phi\left(\tau_{i}, x_{\phi}\left(\tau_{i}, \xi\right)\right)\right) .
\end{aligned}
$$


By (11), (12), and Lemma 1, we have

$$
\begin{aligned}
\left\|T(t, s)^{-1} Q(t) \phi\left(t, x_{\phi}(t, \xi)\right)\right\| & \leq \frac{D^{2}}{1-2 D(M+\Upsilon)} e^{-v(\rho(t)-\rho(s))+a \rho(t)} e^{\mu(\rho(t)-\rho(s))+a \rho(s)}\|\xi\| \\
& =\frac{D^{2}}{1-2 D(M+\Upsilon)} e^{(\mu-v+a)(\rho(t)-\rho(s))+2 a \rho(s)}\|\xi\| .
\end{aligned}
$$

Letting $t \rightarrow \infty$ in (47), it follows from (48) and (17) that (42) holds for every $s \geq 0, \xi \in E(s)$, and $t \geq s$.

If (42) holds for each $s \geq 0, \xi \in E(s)$, and $t \geq s$, we have

$$
\begin{aligned}
T(t, s) Q(s) \phi(s, \xi)+\int_{s}^{t} T(t, \tau) Q(\tau) f\left(\tau, x_{\phi}(\tau, \xi), \phi\left(\tau, x_{\phi}(\tau, \xi)\right)\right) d \tau \\
+\sum_{s \leq \tau_{i}<t} T\left(t, \tau_{i}^{+}\right) Q\left(\tau_{i}^{+}\right) g_{i}\left(\tau_{i}, x_{\phi}\left(\tau_{i}, \xi\right), \phi\left(\tau_{i}, x_{\phi}\left(\tau_{i}, \xi\right)\right)\right) \\
=-\int_{t}^{\infty} T(\tau, t)^{-1} Q(\tau) f\left(\tau, x_{\phi}(\tau, \xi), \phi\left(\tau, x_{\phi}(\tau, \xi)\right)\right) d \tau \\
-\sum_{t \leq \tau_{i}<\infty} T\left(\tau_{i}^{+}, t\right)^{-1} Q\left(\tau_{i}^{+}\right) g_{i}\left(\tau_{i}, x_{\phi}\left(\tau_{i}, \xi\right), \phi\left(\tau_{i}, x_{\phi}\left(\tau_{i}, \xi\right)\right)\right) .
\end{aligned}
$$

Replacing $(s, \xi)$ by $\left(t, x_{\phi}(\tau, \xi)\right)$ in $(36)$, we have

$$
\begin{aligned}
\phi\left(t, x_{\phi}(\tau, \xi)\right)= & -\int_{t}^{\infty} T(\tau, t)^{-1} Q(\tau) f\left(\tau, x_{\phi}(\tau, \xi), \phi\left(\tau, x_{\phi}(\tau, \xi)\right)\right) d \tau \\
& -\sum_{t \leq \tau_{i}<\infty} T\left(\tau_{i}^{+}, t\right)^{-1} Q\left(\tau_{i}^{+}\right) g_{i}\left(\tau_{i}, x_{\phi}\left(\tau_{i}, \xi\right), \phi\left(\tau_{i}, x_{\phi}\left(\tau_{i}, \xi\right)\right)\right) .
\end{aligned}
$$

From (49) and (50), we see that (41) holds for $s \geq 0, \xi \in E(s)$.

Lemma 6 There exists a unique function $\phi \in \Phi$ such that (42) holds for every $s \geq 0, \xi \in$ $E(s)$.

Proof We consider the operator $\widehat{J}$ defined for each $\phi \in \Phi$ by

$$
\begin{aligned}
\widehat{J} \phi)(s, \xi)= & -\int_{s}^{\infty} T(\tau, s)^{-1} Q(\tau) f\left(\tau, x_{\phi}(\tau, \xi), \phi\left(\tau, x_{\phi}(\tau, \xi)\right)\right) d \tau \\
& -\sum_{s \leq \tau_{i}} T\left(\tau_{i}^{+}, s\right)^{-1} Q\left(\tau_{i}^{+}\right) g_{i}\left(\tau_{i}, x_{\phi}\left(\tau_{i}, \xi\right), \phi\left(\tau_{i}, x_{\phi}\left(\tau_{i}, \xi\right)\right)\right),
\end{aligned}
$$

for each $s \geq 0, \xi \in E(s)$. When $\xi=0$, we have $x_{\phi}(\tau, \xi)=0$, then $\left.\widehat{J} \phi\right)(s, 0)=0$. By $\left(\mathrm{H}_{1}\right)$ and Lemma 3, we have

$$
\begin{aligned}
& \left\|f\left(\tau, x_{\phi}(\tau, \xi), \phi\left(\tau, x_{\phi}(\tau, \xi)\right)\right)-f\left(\tau, x_{\phi}(\tau, \bar{\xi}), \phi\left(\tau, x_{\phi}(\tau, \bar{\xi})\right)\right)\right\| \\
& \quad \leq 2 D e^{2 D M} b(\tau) e^{(\mu+\gamma)(\rho(\tau)-\rho(s))+(a+\gamma) \rho(s)}\|\xi-\bar{\xi}\|,
\end{aligned}
$$


and for $i=1,2, \ldots$

$$
\begin{aligned}
& \left\|g_{i}\left(\tau_{i}, x_{\phi}\left(\tau_{i}, \xi\right), \phi\left(\tau_{i}, x_{\phi}\left(\tau_{i}, \xi\right)\right)\right)-g_{i}\left(\tau_{i}, x_{\phi}\left(\tau_{i}, \bar{\xi}\right), \phi\left(\tau_{i}, x_{\phi}\left(\tau_{i}, \bar{\xi}\right)\right)\right)\right\| \\
& \quad \leq 2 D e^{2 D M} c\left(\tau_{i}\right) e^{(\mu+\gamma)\left(\rho\left(\tau_{i}\right)-\rho(s)\right)+(a+\gamma) \rho(s)}\|\xi-\bar{\xi}\| .
\end{aligned}
$$

By (51)-(53), (11), and $\left(\mathrm{H}_{5}\right)-\left(\mathrm{H}_{6}\right)$, for every $s \geq 0, \xi \in E(s)$, we see that

$$
\begin{aligned}
\|(J \phi)(s, \xi)-(J \phi)(s, \bar{\xi})\| \\
\leq \int_{s}^{\infty}\left\|T(\tau, s)^{-1} Q(\tau)\right\| \| f\left(\tau, x_{\phi}(\tau, \xi), \phi\left(\tau, x_{\phi}(\tau, \xi)\right)\right) \\
\quad-f\left(\tau, x_{\phi}(\tau, \bar{\xi}), \phi\left(\tau, x_{\phi}(\tau, \bar{\xi})\right)\right) \| d \tau \\
\quad+\sum_{s \leq \tau_{i}}\left\|T\left(\tau_{i}^{+}, s\right)^{-1} Q\left(\tau_{i}^{+}\right)\right\| \| g_{i}\left(\tau_{i}, x_{\phi}\left(\tau_{i}, \xi\right), \phi\left(\tau_{i}, x_{\phi}\left(\tau_{i}, \xi\right)\right)\right) \\
\quad-g_{i}\left(\tau_{i}, x_{\phi}\left(\tau_{i}, \bar{\xi}\right), \phi\left(\tau_{i}, x_{\phi}\left(\tau_{i}, \bar{\xi}\right)\right)\right) \| \\
\leq 2 D^{2} e^{2 D M}\|\xi-\bar{\xi}\| e^{(a+v-\mu) \rho(s)} \int_{s}^{\infty} b(\tau) e^{(\mu-v+a+\gamma) \rho(\tau)} d \tau \\
\quad+2 D^{2} e^{2 D M}\|\xi-\bar{\xi}\| e^{(a+v-\mu) \rho(s)} \sum_{s \leq \tau_{i}} c\left(\tau_{i}\right) e^{(\mu-v+a+\gamma) \rho\left(\tau_{i}\right)} \\
\leq 2 D^{2} e^{2 D M}(\bar{M}+\bar{\Upsilon})\|\xi-\bar{\xi}\| .
\end{aligned}
$$

It follows from (18) that

$$
\|(J \phi)(s, \xi)-(J \phi)(s, \xi)\| \leq\|\xi-\bar{\xi}\|,
$$

for every $s \geq 0, \xi \in E(s)$, which implies that $J(\Phi) \subset \Phi$.

Given $\phi, \psi \in \Phi, \xi \in E(s)$, by (36), (37), Lemma 1, and Lemma 4, we have

$$
\begin{aligned}
&\|(J \phi)(s, \xi)-(J \psi)(s, \xi)\| \\
& \leq \int_{s}^{\infty}\left\|T(\tau, s)^{-1} Q(\tau)\right\| \| f\left(\tau, x_{\phi}(\tau, \xi), \phi\left(\tau, x_{\phi}(\tau, \xi)\right)\right) \\
& \quad-f\left(\tau, x_{\psi}(\tau, \xi), \psi\left(\tau, x_{\psi}(\tau, \xi)\right)\right) \| d \tau \\
& \quad+\sum_{s \leq \tau_{i}}\left\|T\left(\tau_{i}^{+}, s\right)^{-1} Q\left(\tau_{i}^{+}\right)\right\| \| g_{i}\left(\tau_{i}, x_{\phi}\left(\tau_{i}, \xi\right), \phi\left(\tau_{i}, x_{\phi}\left(\tau_{i}, \xi\right)\right)\right) \\
& \quad-g_{i}\left(\tau_{i}, x_{\psi}\left(\tau_{i}, \xi\right), \psi\left(\tau_{i}, x_{\psi}\left(\tau_{i}, \xi\right)\right)\right) \| \\
& \leq {\left[\frac{D^{2}}{1-2 D(M+\Upsilon)}+\frac{2 D^{2} e^{2 D M}(M+\Upsilon)}{1-2 D(M+\Upsilon)}\right]\|\xi\| d(\phi, \psi) e^{(a+\nu-\mu) \rho(s)} } \\
& \times\left[\int_{s}^{\infty} e^{(\mu-v+a+\gamma) \rho(\tau)} b(\tau) d \tau+\sum \sum_{s \leq \tau_{i}} c\left(\tau_{i}\right) e^{(\mu-v+a+\gamma) \rho\left(\tau_{i}\right)}\right] \\
& \leq {\left[\frac{D^{2}}{1-2 D(M+\Upsilon)}+\frac{2 D^{2} e^{2 D M}(M+\Upsilon)}{1-2 D(M+\Upsilon)}\right](\bar{M}+\bar{\Upsilon})\|\xi\| d(\phi, \psi) . }
\end{aligned}
$$


Thus, the operator $J: \Phi \rightarrow \Phi$ becomes a contraction. Therefore, for each $\xi \in E(s)$, there exists a unique function $\phi$ satisfying (21). By Lemma 3, we have

$$
\begin{aligned}
& \left\|\Psi_{\epsilon}(s, \xi, \phi(s, \xi))-\Psi_{t}(s, \bar{\xi}, \phi(s, \bar{\xi}))\right\| \\
& \quad=\left\|\left(t, x_{\phi}(t, \xi), \phi\left(s, x_{\phi}(t, \xi)\right)\right)-\left(t, x_{\phi}(t, \bar{\xi}), \phi\left(s, x_{\phi}(t, \bar{\xi})\right)\right)\right\| \\
& \quad \leq 2\left\|x_{\phi}(t, \xi)-x_{\phi}(t, \bar{\xi})\right\| \leq 2 D e^{2 D M} e^{(\mu+\gamma)(\rho(t)-\rho(s))+a \rho(s)}\|\xi-\bar{\xi}\| .
\end{aligned}
$$

This completes the proof of Theorem 2 .

\section{Stable manifolds under perturbations}

This section is to see how the manifolds vary with the perturbations. Let $\Omega$ be space of the function $f: R^{+} \times R^{n} \rightarrow R^{n}$ and $\bar{\Omega}$ be the space of all sequences of function $g=g_{i}$ : $R^{+} \times R^{n} \rightarrow R^{n}$. We define a metric by

$$
\|f-\bar{f}\|_{*}=\sup \left\{\frac{\|f(t, x)-\bar{f}(t, x)\|}{\|x\|}, t \geq 0, x \in R^{n} \backslash\{0\}\right\}
$$

and

$$
\|g-\bar{g}\|_{* *}=\sup _{i \geq 1} \sup \left\{\frac{\left\|g_{i}\left(\tau_{i}, x\right)-\bar{g}_{i}\left(\tau_{i}, x\right)\right\|}{\|x\|}, \tau_{i} \geq 0, x \in R^{n} \backslash\{0\}\right\} .
$$

We also assume that the following conditions hold:

$\left(\mathrm{H}_{7}\right) \quad 0<\widehat{M}=\sup _{s \geq 0}\left\{e^{a+\nu-\mu} \int_{s}^{\infty} e^{(\mu-\nu+a) \rho(\tau)} d \tau\right\}<\infty$;

$\left(\mathrm{H}_{8}\right) \quad 0<\widehat{\Upsilon}=\sup _{s \geq 0}\left\{e^{a+\nu-\mu} \sum_{\tau_{i} \geq s} e^{(\mu-v+a) \rho\left(\tau_{i}\right)}\right\}<\infty$.

Theorem 3 Assume that (1) admits a $\rho$-nonuniform exponential dichotomy. If $\left(\mathrm{H}_{1}\right)-\left(\mathrm{H}_{8}\right)$, (16)-(19) hold, then

$$
\|\varphi-\psi\| \leq D_{1}\|f-\bar{f}\|_{*}+D_{2}\|g-\bar{g}\|_{* *}
$$

for every $f, \bar{f} \in \Omega$ and $g, \bar{g} \in \bar{\Omega}$, where $\phi, \psi \in \Phi$ are the functions given by Theorem 2 corresponding to the perturbations $f, g$ and $\bar{f}, \bar{g}, D_{1}=\frac{2 D^{2} \widehat{M}}{[1-2 D(M+\Upsilon)]\left\{1-\left[\frac{D^{2}}{1-2 D(M+\Upsilon)}+\frac{2 D^{2} e^{2 D M}(M+\Upsilon)}{1-2 D(M+\Upsilon)}\right](\bar{M}+\bar{\Upsilon})\right\}}$, $D_{2}=\frac{2 D^{2} \widehat{\Upsilon}}{[1-2 D(M+\Upsilon)]\left\{1-\left[\frac{D^{2}}{1-2 D(M+\Upsilon)}+\frac{2 D^{2} e^{2 D M}(M+\Upsilon)}{1-2 D(M+\Upsilon)}\right](\bar{M}+\bar{\Upsilon})\right\}}$.

Proof For every $\xi \in E(s)$, by (36), (37), (58), (59), and Lemma 4, we have

$$
\begin{aligned}
q_{5}(\tau)= & \left\|f\left(\tau, x_{\phi}(\tau, \xi), \phi\left(\tau, x_{\phi}(\tau, \xi)\right)\right)-\bar{f}\left(\tau, x_{\psi}(\tau, \xi), \psi\left(\tau, x_{\psi}(\tau, \xi)\right)\right)\right\| \\
\leq & \left\|f\left(\tau, x_{\phi}(\tau, \xi), \phi\left(\tau, x_{\phi}(\tau, \xi)\right)\right)-\bar{f}\left(\tau, x_{\phi}(\tau, \xi), \phi\left(\tau, x_{\phi}(\tau, \xi)\right)\right)\right\| \\
& +\left\|\bar{f}\left(\tau, x_{\phi}(\tau, \xi), \phi\left(\tau, x_{\phi}(\tau, \xi)\right)\right)-\bar{f}\left(\tau, x_{\psi}(\tau, \xi), \psi\left(\tau, x_{\psi}(\tau, \xi)\right)\right)\right\| \\
\leq & \frac{2 D}{1-2 D(M+\Upsilon)} e^{\mu(\rho(\tau)-\rho(s))+a \rho(s)}\|f-\bar{f}\|_{*}+\left[\frac{D}{1-2 D(M+\Upsilon)}\right. \\
& \left.+\frac{2 D e^{2 D M}(M+\Upsilon)}{1-2 D(M+\Upsilon)}\right] b(\tau) e^{(\mu+\gamma)(\rho(\tau)-\rho(s))+(a+\gamma) \rho(s)}\|\xi\|\|\phi-\psi\|,
\end{aligned}
$$


and for $i=1,2, \ldots$

$$
\begin{aligned}
q_{6}\left(\tau_{i}\right)= & \left\|g_{i}\left(\tau_{i}, x_{\phi}\left(\tau_{i}, \xi\right), \phi\left(\tau_{i}, x_{\phi}\left(\tau_{i}, \xi\right)\right)\right)-\bar{g}_{i}\left(\tau_{i}, x_{\psi}\left(\tau_{i}, \xi\right), \psi\left(\tau_{i}, x_{\psi}\left(\tau_{i}, \xi\right)\right)\right)\right\| \\
\leq & \frac{2 D}{1-2 D(M+\Upsilon)} e^{\mu\left(\rho\left(\tau_{i}\right)-\rho(s)\right)+a \rho(s)}\|g-\bar{g}\|_{* *}+\left[\frac{D}{1-2 D(M+\Upsilon)}\right. \\
& \left.+\frac{2 D e^{2 D M}(M+\Upsilon)}{1-2 D(M+\Upsilon)}\right] c\left(\tau_{i}\right) e^{(\mu+\gamma)\left(\rho\left(\tau_{i}\right)-\rho(s)\right)+(a+\gamma) \rho(s)}\|\xi\|\|\phi-\psi\| .
\end{aligned}
$$

It follows from (11), (42), (61), and (62) that

$$
\begin{aligned}
&\|\phi(s, \xi)-\psi(s, \xi)\| \\
&=\int_{s}^{\infty}\left\|T(\tau, s)^{-1} Q(\tau)\right\| q_{5}(\tau) d \tau+\sum_{s \leq \tau_{i}}\left\|T\left(\tau_{i}^{+}, s\right)^{-1} Q\left(\tau_{i}^{+}\right)\right\| q_{6}\left(\tau_{i}\right) \\
& \leq \frac{2 D^{2}}{1-2 D(M+\Upsilon)}\|f-\bar{f}\|_{*} e^{(a+\nu-\mu) \rho(s)} \int_{s}^{\infty} e^{(\mu-v+a) \rho(\tau)} d \tau \\
&+\frac{2 D^{2}}{1-2 D(M+\Upsilon)}\|g-\bar{g}\|_{* *} e^{(a+\nu-\mu) \rho(s)} \sum_{s \leq \tau_{i}} e^{(\mu-v+a) \rho\left(\tau_{i}\right)} \\
&+\left\{\left[\frac{D^{2}}{1-2 D(M+\Upsilon)}+\frac{2 D^{2} e^{2 D M}(M+\Upsilon)}{1-2 D(M+\Upsilon)}\right] e^{(a+\nu-\mu) \rho(s)} \int_{s}^{\infty} b(\tau) e^{(\mu-v+a+\gamma) \rho(\tau)} d \tau\right. \\
&+\left[\frac{D^{2}}{1-2 D(M+\Upsilon)}+\frac{2 D^{2} e^{2 D M}(M+\Upsilon)}{1-2 D(M+\Upsilon)}\right] e^{(a+\nu-\mu) \rho(s)} \\
&\left.\times \sum_{s \leq \tau_{i}} c\left(\tau_{i}\right) e^{(\mu-v+a+\gamma) \rho\left(\tau_{i}\right)}\right\}\|\xi\|\|\phi-\psi\| \\
& \leq \frac{2 D^{2} \widehat{M}}{1-2 D(M+\Upsilon)}\|f-\bar{f}\|_{*}+\frac{2 D^{2} \widehat{\Upsilon}}{1-2 D(M+\Upsilon)}\|g-\bar{g}\|_{* *} \\
&+\left[\frac{D^{2}}{1-2 D(M+\Upsilon)}+\frac{2 D^{2} e^{2 D M}(M+\Upsilon)}{1-2 D(M+\Upsilon)}\right](\bar{M}+\bar{\Upsilon})\|\xi\|\|\phi-\psi\|
\end{aligned}
$$

which implies that (60) holds.

\section{Example}

In this section, we provide an example to demonstrate the derived results. Consider the impulsive system

$$
\left\{\begin{array}{lrl}
x^{\prime} & =\left[2 \tilde{\mu} t+\bar{a} t\left(t^{2} \cos t^{2}-1\right)\right] x+b(t) x, & \left.\Delta x\right|_{t=\tau_{i}}=b_{i} x\left(\tau_{i}\right)+c\left(\tau_{i}\right) x\left(\tau_{i}\right) \\
y^{\prime} & =\left[2 \widetilde{v} t+\bar{a} t\left(t^{2} \cos t^{2}-1\right)\right] y+\frac{b(t) y}{1+y^{2}}, & \left.\Delta y\right|_{t=\tau_{i}}=b_{i} y\left(\tau_{i}\right)+\frac{c\left(\tau_{i}\right) y\left(\tau_{i}\right)}{1+y^{2}\left(\tau_{i}\right)}
\end{array}\right.
$$

where $\tilde{\mu}<0, \widetilde{v}>0, \bar{a}>0, b_{i} \geq 0$ and $b(t)>0, c\left(\tau_{i}\right)>0, i=1,2, \ldots$. Assume that there exists $\delta>0$ such that $\frac{\ln \left(1+b_{i}\right)}{\tau_{i}^{2}-\tau_{i-1}^{2}} \leq \delta, i=1,2, \ldots$. Setting $P(t)(x, y)=(x, 0)$ and $Q(t)(x, y)=(0, y)$, we have

$$
\begin{aligned}
& \|T(t, s) P(s)\| \leq e^{\bar{a}} e^{\widetilde{\mu}\left(t^{2}-s^{2}\right)+a s^{2}} \prod_{s \leq \tau_{i}<t}\left(1+b_{i}\right) \leq e^{\bar{a}} e^{(\widetilde{\mu}+\delta)\left(t^{2}-s^{2}\right)+(a+\delta) s^{2}}, \\
& \left\|T(t, s)^{-1} Q(s)\right\| \leq e^{\bar{a}} e^{-\widetilde{\nu}\left(t^{2}-s^{2}\right)+a t^{2}} \prod_{s \leq \tau_{i}<t}\left(1+b_{i}\right) \leq e^{\bar{a}} e^{-\widetilde{\nu}\left(t^{2}-s^{2}\right)+(a+\delta) t^{2}} .
\end{aligned}
$$


This shows that the linear part of (64) admits a $\rho$-nonuniform dichotomy with

$$
\rho(t)=t^{2}, \quad \mu=\tilde{\mu}+\delta, \quad v=\widetilde{v}, \quad a=\bar{a}+\delta, \quad D=e^{\bar{a}} .
$$

We conclude that (64) has a stable invariant manifold provided that $\left(\mathrm{H}_{1}\right)-\left(\mathrm{H}_{6}\right)$ and (16)-

(19) hold.

\section{Competing interests}

The author declares that he has no competing interests.

Author's contributions

The author contributed to the manuscript. The author read and approved the final manuscript.

\section{Acknowledgements}

The author would like to thank the referee and the associate editor for their very helpful suggestions.

Received: 22 November 2014 Accepted: 28 May 2015 Published online: 17 July 2015

\section{References}

1. Lakshmikantham, V, Bainov, DD, Simeonov, PS: Theory of Impulsive Ordinary Differential Equations. World Scientific, Singapore (1989)

2. Samoilenko, AM, Perestyuk, NA: Impulsive Differential Equations. World Scientific, Singapore (1995)

3. Perron, O: Die Stabilitätsfrage bei Differentialgleichungen. Math. Z. 32, 703-728 (1930)

4. Massera, J, Schäffer, J: Linear Differential Equations and Function Spaces. Pure Appl. Math., vol. 21. Academic Press, New York (1966)

5. Dale'ckiĭ, J, Kreĭn, M: Stability of Solutions of Differential Equations in Banach Space. Trans. Math. Monogr., vol. 43. Am. Math. Soc., Providence (1974)

6. Coppel, W: Dichotomies in Stability Theory. Lecture Notes in Math., vol. 629. Springer, Berlin (1978)

7. Sacker, R, Sell, G: Existence of dichotomies and invariant splittings for linear differential systems. I. J. Differ. Equ. 15 429-458 (1974)

8. Sacker, R, Sell, G: Existence of dichotomies and invariant splittings for linear differential systems. II. J. Differ. Equ. 22, 478-496 (1976)

9. Sacker, R, Sell, G: Existence of dichotomies and invariant splittings for linear differential systems. III. J. Differ. Equ. 22, 497-522 (1976)

10. Sacker, R, Sell, G: Dichotomies for linear evolutionary equations in Banach spaces. J. Differ. Equ. 113, 17-67 (1994)

11. Barreira, L, Valls, C: Stability of Nonautonomous Differential Equations. Lecture Notes in Math., vol. 1926. Springer, Berlin (2008)

12. Bento, AJG, Lupa, N, Megan, M, Silva, CM: Integral conditions for nonuniform-dichotomy. arXiv:1405.2946 [math. DS]

13. Oseledets, V: A multiplicative ergodic theorem. Liapunov characteristic numbers for dynamical systems. Trans. Mosc. Math. Soc. 19, 197-221 (1968)

14. Barreira, L, Pesin, Y: Nonuniform Hyperbolicity. Encyclopedia Math. Appl., vol. 115. Cambridge University Press, Cambridge (2007)

15. Massera, J, Schäffer, J: Linear differential equations and functional analysis. I. Ann. Math. (2) 67, 517-573 (1958)

16. Barreira, L, Valls, C: Stable manifolds for nonautonomous equations without exponential dichotomy. J. Differ. Equ. 221, 58-90 (2006)

17. Chow, SN, Leiva, H: Existence and roughness of the exponential dichotomy for skew-product semiflow in Banach spaces. J. Differ. Equ. 120, 429-477 (1995)

18. Pliss, V, Sell, G: Robustness of exponential dichotomies in infinite-dimensional dynamical systems. J. Dyn. Differ. Equ 11, 471-513 (1999)

19. Barreira, L, Valls, C: Lyapunov regularity of impulsive differential equations. J. Differ. Equ. 249, 1596-1619 (2010)

20. Barreira, L, Valls, C: Stable manifolds for impulsive equations under nonuniform hyperbolicity. J. Dyn. Differ. Equ. 22, 761-785 (2010)

21. Barreira, L, Fan, M, Valls, C, Zhang, J: Invariant manifolds for impulsive equations and nonuniform polynomial dichotomies. J. Stat. Phys. 141, 179-200 (2010)

22. Zhang, J, Chang, $X$, Wang, J: Existence and robustness of nonuniform $(h, k, \mu, v)$-dichotomies for nonautonomous impulsive differential equations. J. Math. Anal. Appl. 400, 710-723 (2013)

23. Barreira, L, Valls, C: Growth rates and nonuniform hyperbolicity. Discrete Contin. Dyn. Syst. 22, 509-528 (2008)

24. Barreira, L, Valls, C: Robustness of general dichotomies. J. Funct. Anal. 257, 464-484 (2009)

25. Barreira, L, Valls, C: Stable invariant manifolds for parabolic dynamics. J. Funct. Anal. 257, 1018-1029 (2009) 